\title{
Limits on axion-photon coupling or on local axion density: Dependence on models of the Milky Way's dark halo
}

\author{
J.V. Sloan a , M. Hotz ${ }^{\mathrm{a}}$, C. Boutan ${ }^{\mathrm{a}}$, R. Bradley ${ }^{\mathrm{c}}$, G. Carosi ${ }^{\mathrm{b}}$, D. Carter ${ }^{\mathrm{b}}$, J. Clarke $^{\mathrm{d}}$, N. \\ Crisosto $^{\text {e }}$, E.J. Daw ${ }^{\mathrm{f}}$, J. Gleason ${ }^{\mathrm{e}}$, J. Hoskins ${ }^{\mathrm{e}}$, R. Khatiwada ${ }^{\mathrm{a}}$, D. Lyapustin ${ }^{\mathrm{a}}$, A. \\ Malagon $^{\text {a }}$, S. O'Kelley ${ }^{\mathrm{d}}$, R.S. Ottens ${ }^{\mathrm{a}}$, L.J Rosenberg ${ }^{\mathrm{a}}$, G. Rybka ${ }^{\mathrm{a}}$, I. Stern ${ }^{\mathrm{e}}$, N.S. \\ Sullivan $^{\mathrm{e}}$, D.B. Tanner ${ }^{\mathrm{e}}$, K. van Bibber ${ }^{\mathrm{d}}$, A. Wagner ${ }^{\mathrm{a}, 1}$, D. Will ${ }^{\mathrm{a}}$ \\ ${ }^{a}$ Center for Experimental Nuclear Physics and Astrophysics $\mathcal{E}$ University of Washington, Seattle WA 98195 \\ ${ }^{b}$ Lawrence Livermore National Laboratory, Livermore CA 94550 \\ ${ }^{c}$ National Radio Astronomy Observatory, Charlottesville VA 22903 \\ ${ }^{d}$ University of California, Berkeley CA 94720 \\ ${ }^{e}$ University of Florida, Gainesville FL 32611 \\ ${ }^{f}$ University of Sheffield, Sheffield UK S10 2TN
}

\begin{abstract}
The $\mu \mathrm{eV}$-scale axion is a compelling cold dark matter candidate. The Axion Dark Matter eXperiment (ADMX) searches for axions by stimulating the decay of galactic dark matter halo axions into detectable microwave photons by their conversion in a resonant cavity permeated by a strong, static magnetic field. The signal depends on properties of the Milky Way's dark matter halo; the choice of halo model has significant implications for the sensitivity of direct detection searches, e.g., ADMX. This paper explores the sensitivity of the data taken by ADMX from 2008 to 2010 to various dark matter halo models. New models for the phase-space distribution of local axions are considered; the analysis demonstrates that certain assumptions about the dark matter halo improve limits on axion-photon coupling. In addition, new ADMX data covering $860-892 \mathrm{MHz}$ are included in the analysis.
\end{abstract}

\section{Introduction}

Experiments and observations have placed limits on axion-photon couplings or on the local axion density. The construction of limits from direct-detection searches requires properties of the Milky Way's dark matter halo. The details of these properties are unknown; therefore, limits are dependent upon the assumption of a halo model. Typically, exclusion limits are generated using the most basic and conservative model: an isothermal distribution. In this paper, models of the Milky Way's dark matter halo with additional structure are used to set limits on axion-photon coupling (or, equivalently on the axion density) using data from the ADMX detector.

${ }^{1}$ Present address: Raytheon BBN Technologies, Cambridge MA 02138 
The present study explores a broader range of N-body motivated halo models than in previous work; the models are introduced in Sec. 3.2 and the corresponding analysis is detailed in Sec.7. Thermalized QCD axion limits generated from $810-860 \mathrm{MHz}$ of this data set have been published in Ref. [1]; the limits from $860-892 \mathrm{MHz}$ are novel. A search for cold flow axions using this data set was published in Ref. [2, 3]; however, the specific models and the analysis differ substantially from the present work.

The results demonstrate ADMX's sensitivity to a variety of halo models and the feasibility of extracting galactic dark matter structure from an axion signal's line shape. At the time of writing, ADMX has begun a data run with unprecedented scan rate and sensitivity. The analysis of the upcoming data will be informed by the findings summarized in this paper and, in the case of discovery, ADMX will be better equipped to interpret cosmological information encoded in the axion signal.

\section{Dark Matter Axions}

\subsection{Dark Matter}

The overall matter and energy composition of the universe is now known with good precision [4]. The majority of the universe's matter, however, has never been observed directly, only inferred gravitationally [5, 6]. This unidentified matter is called dark matter, and its nature is one of the most conspicuous unanswered questions in science today. While the total mass of dark matter within the Milky Way is well known, its distribution is not; models predict a wide range of dark matter halos. The properties of the local dark matter halo determine the sensitivity of dark matter direct detection experiments such as ADMX; therefore, implications of the range of models should be considered. The models selected for this work are discussed in Sec. 3.2

The axion is a compelling cold dark matter candidate due to feeble coupling to ordinary matter, non-relativistic energies, and early universe phenomenology consistent with dark matter cosmology [7, 8, 9, 10, 11, 12, 13, 14]. The axion originated as a consequence of the Peccei-Quinn solution to the strong CP problem in quantum chromodynamics [15, 16, 17].

\subsection{The $Q C D$ Axion}

The Lagrangian describing quantum chromodynamics naturally includes a CPsymmetry violating term

$$
\mathcal{L}_{\theta}=\bar{\theta}\left(\frac{\alpha_{s}}{8 \pi}\right) G^{\mu v a} \tilde{G}_{\mu v}^{a}
$$

where $G^{a \mu \nu}$ is the gluon field strength tensor, $\tilde{G}_{\mu \nu}^{a}$ its dual, $\alpha_{s}$ is the strong coupling constant, and $\bar{\theta}=\theta+\arg \operatorname{det} M$, where $\theta$ is a gauge-dependent free parameter and $M$ is the quark mass matrix from weak physics. The term $\mathcal{L}_{\theta}$ is a total derivative and has no effect on perturbative calculation but does produces parity-symmetry violation due to non-perturbative QCD effects.

The parameter $\bar{\theta}$ contains contributions from disparate sectors of physics; therefore, asserting that it takes a very small or zero value is making an unacceptable fine-tuning argument [18]. However, experiments have constrained $\bar{\theta}$ to a very small value. The 
strongest limit comes from measurements of the neutron electric dipole made by the nEDM experiment. The neutron electric dipole moment has a value of $\bar{\theta} \times 10^{-16} \mathrm{e}-\mathrm{cm}$; searches by nEDM have set an upper bound of $2.9 \times 10^{-26} \mathrm{e}-\mathrm{cm}$, corresponding to a limit of $\bar{\theta}<10^{-10}[19]$. This result suggests that the standard model is extraordinarily finely-tuned in $\bar{\theta}$.

The Peccei-Quinn mechanism solves this fine-tuning strong $\mathrm{CP}$ problem by means of a new, hidden $U_{A}(1)$ symmetry, $U_{A}(1)_{P Q}$, that is broken at some scale, $f_{a}$, and dynamically drives $\langle\bar{\theta}\rangle$ to zero [15]. This spontaneously broken symmetry leads to a new pseudoscalar Nambu Goldstone boson, named the axion [16, 17]. Consequently,

$$
\bar{\theta} \rightarrow\left(\bar{\theta}-\frac{\phi_{a}}{f_{a}}\right)
$$

where $\phi_{a}$ is the axion field and $f_{a}$ is the axion decay constant.

The axion-photon coupling strength, $g_{a \gamma \gamma}$, and mass, $m_{a}$, follow $m_{a} \propto g_{a \gamma \gamma} \propto 1 / f_{a}$. The chargeless axion couples to two photons via anomalies. In general, the predicted coupling constant ranges between $0.1 m_{a} \mathrm{GeV}^{-2}<\left|g_{a \gamma \gamma}\right|<0.43 m_{a} \mathrm{GeV}^{-2}$ for the DFSZ axions (color anomalies only, [12, 13]) and KSVZ axion (electromagnetic and color anomalies, [10, 11]). $g_{a \gamma \gamma}$ is determined by a model-dependent constant, $g_{\gamma}$, according to the relationship $g_{a \gamma \gamma}=\frac{\alpha g_{\gamma}}{\pi f_{a}}$, where $\alpha$ is the fine structure constant. Typical values of $g_{\gamma}$ are 0.36 for DFSZ [12, 13] and -0.97 for KSVZ [10, 11]. In this paper, we primarily focus on KSVZ and DFSZ axions, and restrict our attention to the standard QCD axion, i.e. the axion that arises from the Peccei-Quinn solution to the strong CP problem.

Existing experimental bounds on the axion-photon coupling and axion mass are shown in Fig. 1. Axions with $m_{a}>16 \mathrm{meV}$ or $g_{a \gamma \gamma}>10^{-10} \mathrm{GeV}^{-1}$ have been excluded by a variety of searches and astronomical observations [18, 20]. In the classic post-inflationary Peccei-Quinn symmetry breaking model, axion masses below the $\mu \mathrm{eV}$ scale produce more dark matter than is observed [18, 20]; however, this bound may be evaded or enhanced in a number of ways. The energy associated with $\langle\bar{\theta}\rangle$ being forced to zero is manifested as primordial axions and could dominate the mass budget of the universe; in fact, the remaining plausible axion mass range implies that if axions exist, then axions are a major constituent of dark matter.

\section{Detecting Dark Matter Axions}

\subsection{Axion Haloscope}

The coupling of axions to ordinary matter is extraordinarily small, thus detecting axions is challenging. One proven method to detect $\mu \mathrm{eV}$-scale axions is the axion haloscope [21]. An axion haloscope consists of a strong static magnetic field inside a microwave resonant cavity. Within the cavity, a reverse Primakoff process occurs: Axions scatter off the virtual photons of the magnetic field and thereby convert into real photons. The conversion rate is enhanced by the cavity resonance when the cavity is tuned to the outgoing microwave photon frequency. The haloscope greatly increases the decay rate of axions from the Milky Way's dark matter halo and measures the resulting power. 


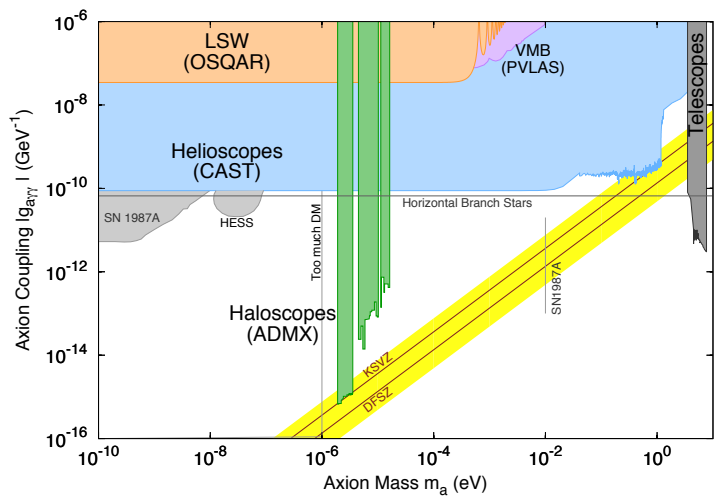

Figure 1: Existing bounds on axion-like particles (ALPs) and QCD axions. The vertical axis represents the axion-photon coupling strength. The horizontal axis represents the axion mass. The KSVZ and DFSZ lines represent benchmark QCD axion models. The yellow band represents plausible QCD axion models from reference [14]. Various experimental, cosmological and astronomical results have produced limits on the axion mass and axion-photon coupling strength [18 20]. Limits generated by axion haloscopes necessarily assume a dark matter halo model; as shown, the limits assume an isothermal distribution with axion density $\rho=0.45 \pm 0.3 \mathrm{GeV} / \mathrm{cm}^{3}$. The possible parameter space of QCD axions is constrained to within the yellow band ranging in mass from about $1 \mu \mathrm{eV}$ to $10 \mathrm{meV}$. An axion with parameters within these bounds may be a major component of the dark matter in the universe.

The power developed in an axion haloscope with typical ADMX parameters is [21]

$$
\begin{aligned}
P_{a}= & \left(1.19 \times 10^{-21} \mathrm{~W}\right)\left(\frac{V}{140 \mathrm{~L}}\right)\left(\frac{B}{7.6 \mathrm{~T}}\right)^{2}\left(f_{n l m}\right) \times \\
& \left(\frac{\rho_{a}}{0.45 \frac{\mathrm{GeV}}{\mathrm{cm}^{3}}}\right)\left(\frac{g_{\gamma}}{-0.97}\right)^{2}\left(\frac{Q}{140000}\right)\left(\frac{v_{a}}{750 \mathrm{MHz}}\right)
\end{aligned}
$$

where $V$ is the cavity volume, $B$ is the magnetic field, $f_{n l m}$ is the form factor of the cavity mode and magnetic field shape (discussed in Sec. 4.2p, $\rho_{a}$ is the local energy density of axions, $g_{\gamma}$ is the model-dependent coupling parameter (defined in Sec. 2.2. $v_{a}$ is the frequency of the photon signal due to axion conversion, and $Q$ is the quality factor of the unloaded cavity. Some of this power is extracted from the cavity by a nearcritically-coupled field probe. The power extracted by the field probe is then amplified and processed by an ultra-low-noise microwave receiver chain which is discussed in Sec. 4.4

For a haloscope with typical ADMX parameters, the expected signal is of order $10^{-22} \mathrm{~W}$; therefore, the principal technical challenge is reducing the system noise. The two primary sources of noise in a haloscope are the cavity walls and the first amplifier in the receiver chain. The walls of the cavity produce a spectrum of thermal blackbody photons (Johnson noise) identical in power spectral density to the white noise generated by a resistor at the same temperature. The first amplifier in the receiver also produces noise; this noise depends on details of the amplifier construction. The total noise power 
within the axion signal bandwidth is

$$
\begin{aligned}
P_{N}= & \left(5.52 \times 10^{-20} \mathrm{~W}\right) \times \\
& \left(\frac{T_{\text {Cavity }}+T_{\text {Amplifier }}}{4 \mathrm{~K}}\right)\left(\frac{b_{a}}{1000 \mathrm{~Hz}}\right)
\end{aligned}
$$

where $T_{\text {Cavity }}$ is the physical temperature of the cavity and $T_{\text {Amplifier }}$ is the Johnsonequivalent noise temperature for the first amplifier in the receiver chain. The halomodel-dependent axion signal bandwidth, $b_{a}$, isdiscussed in Sec. 3.2

The sensitivity of an axion haloscope is characterized by the signal-to-noise ratio (SNR), given by the ideal radiometer equation [22]

$$
S N R=\frac{P_{a}}{2 P_{N}} \sqrt{b_{a} t_{\text {int }}}
$$

where $P_{a}$ is from Eq. 3, $P_{N}$ is the noise power, and $t_{\text {int }}$ is the integration time. At critical coupling, $\frac{1}{2}$ of the power is extracted from the cavity. To detect the axion signal with good confidence, the SNR needs to be at least 10. Because the axion mass is not known a priori, an axion haloscope is tuned to search for a range of possible axion masses. The scan rate for an axion haloscope with typical parameters, for SNR of 10, is

$$
\begin{aligned}
R= & \left(12.28 \frac{\mathrm{Hz}}{s}\right)\left(\frac{v_{a}}{Q} \frac{2}{b_{a}}\right)\left(\frac{P_{a}}{2} \frac{1}{1.19 \times 10^{-21} \mathrm{~W}}\right)^{2} \times \\
& \left(\frac{4 \mathrm{~K}}{T_{\text {Cavity }}+T_{\text {Amplifier }}}\right)^{2}
\end{aligned}
$$

where $2 v_{a} / Q$ is the bandwidth of the critically coupled cavity.

\subsection{Dark Matter Halo Models Selected for Analysis}

The signal produced in an axion haloscope and the sensitivity of an axion haloscope are dependent on various properties of the local dark matter halo. The parameters in Equations 3- 5 that are determined by the dark matter halo are $v_{a}, \rho$, and $b_{a}$. The frequency of the detectable photon, $v_{a}$, corresponds to the sum of the rest mass and kinetic energy of the incoming axion. The width of the signal in frequency space, $b_{a}$, is directly determined by the velocity dispersion of the axion halo.

Assumptions of the properties of the local dark matter halo are a necessary input to construct exclusion limits on the existence of dark matter particles. Conversely, once an axion signal is detected, its line shape encodes information about the local distribution of dark matter. Four models representing a wide range of structure formation were selected for use in the present analysis. Here the models are described qualitatively; the specific values used for analysis are stated in Sec. 7

\subsubsection{Isothermal Model}

In the isothermal model, dark matter is a collisionless gas of uniform temperature and, therefore, uniform velocity dispersion [23, 24]. The signal resulting from an isothermal distribution of axions at our location yields a signal that is approximately 
a Maxwell-Boltzman distribution with width proportional to the dark matter velocity dispersion [25]. The isothermal distribution is the simplest model; more sophisticated models typically include an isothermal distribution augmented with additional structure. Sensitivity to the isothermal distribution model, therefore, can be considered baseline sensitivity to the local dark matter halo.

\subsubsection{Dark Disk Model}

According to the dark disk model, the dark matter halo is the combination of an isothermal distribution and a thin, cold disk of dark matter from recent accretion of dark matter subhalos, as described in [26, 27]. For example, a dark disk could have been formed by a merger of the Milky Way with a dwarf galaxy. The signal of this model is the sum of the isothermal signal and a narrower peak from the colder disk.

\subsubsection{Late Infall Model}

As dark matter falls into galaxies during galaxy formation, the most recently infalling dark matter may not yet have thermalized with the rest of the halo. Due to the resulting low velocity dispersion, a dark matter "flow" produces a signal that is very narrow in frequency space [28, 25]. However, the relative velocities of the Earth and the dark matter flow result in a signal that shifts in frequency with time.

\subsubsection{Caustic Model}

Some late infall models predict that dark matter overdensities, in the form of caustics, occur where the velocity of many dark matter particles is close to zero and the particles coalesce [29, 25]. If the Earth is near one of these dark matter caustics, the local dark matter velocity dispersion would be similarly small as for general late infall models but with significantly greater local dark matter density. Consequently, the existence of dark matter caustics potentially greatly increases an axion haloscope's sensitivity [30].

\section{ADMX}

The Axion Dark Matter eXperiment (ADMX) is an axion haloscope that has searched for dark matter axions since 1995. Fig. 2 shows a cutaway of the hardware of ADMX in 2008-2010, including the vertically-oriented main magnet and the removable experimental insert, that is housed in a 3.4-m tall cryostat. The experimental insert is comprised of the cavity, cryogenic components, cryogenic amplifiers, and supporting thermal and mechanical infrastructure. For the duration of the work described in this paper, ADMX was located at Lawrence Livermore National Laboratory in Livermore, CA. The experimental apparatus is described more fully in Ref. [31] and the receiver's design and performance are detailed in Ref. [32].

\subsection{Magnet}

The ADMX main magnet produces the field necessary for the conversion of dark matter axions into photons. The superconducting coil is a $1.12-\mathrm{m}$ tall solenoid with a $0.6-\mathrm{m}$ inner diameter. It is made of niobium-titanium winding, and has an inductance of $534 \mathrm{H}$. The maximum field is $8.5 \mathrm{~T}$. 


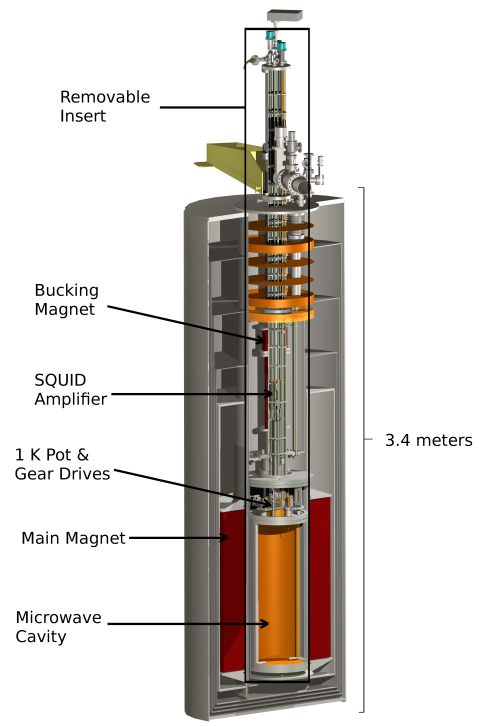

Figure 2: Cutaway view of the ADMX cryostat.

\subsection{Cavity}

Axions interact with the magnetic field and convert into photons within the ADMX resonant cavity, a 140-L copper-plated and annealed stainless-steel cylinder. The cylinder is made of a tube forming the cavity walls and two removable end caps. The end caps form a low-resistance connection to the walls via a knife-edge on the walls pressing firmly into the end plates. The loaded quality factor, $\mathrm{Q}$, of the cavity varied substantially during data taking; a range of typical values is approximately 3,500-40, 000 .

The power developed in the cavity by the axion-to-photon conversion given by Eq. 3 depends on the form factor, $f_{n l m}$, of the cavity mode, which parametrizes the overlap of the static magnetic field with a cavity mode electric field. The form factor is defined as

$$
f_{n l m} \equiv \frac{\left(\int_{V} d V \mathbf{E}(\mathbf{x}) \cdot \mathbf{B}(\mathbf{x})\right)^{2}}{\left(\int_{V} d V \epsilon_{r} E^{2}(\mathbf{x})\right)\left(\int_{V} d V B^{2}(\mathbf{x})\right)}
$$

where $\mathbf{E}(\mathbf{x}, t)$ is the electric field of the $n l m$ cavity mode ${ }^{2} V$ is the cavity volume, $\epsilon_{r}$ is the relative permittivity within the cavity, and $\mathbf{B}$ is the applied magnetic field. The magnetic field is coaxial with the cavity. For this configuration, $f_{n l m} \neq 0$ only for specific transverse-magnetic modes, $\mathrm{TM}_{0 l 0}$ modes, and is maximized by the $\mathrm{TM}_{010}$ mode. The limits presented in this paper were generated by data taken using the $\mathrm{TM}_{010}$ mode.

\footnotetext{
${ }^{2}$ The "nlm" nomenclature is strictly appropriate only for an empty cylindrical (or rectangular) cavity; the modes that ADMX calls "TM $\mathrm{TM}_{0 l 0}$ " can be continuously connected to proper $\mathrm{TM}_{0 l 0}$ modes by reducing the tuning rods' diameter incrementally to zero in simulation.
} 


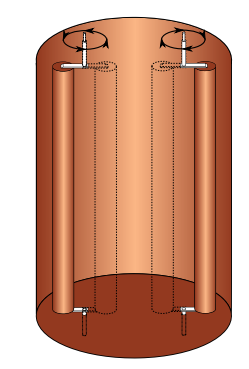

Figure 3: A sketch illustrating the path of the tuning rods as they are moved within the cavity.

To tune the $\mathrm{TM}_{010}$ mode, two copper axial tuning rods are mounted on rotating alumina supports inside the cavity. The path of the rods is illustrated in Fig. 3. Rotating the rods from near the wall of the cavity to near the center tunes the $\mathrm{TM}_{010}$ mode from appoximately $500 \mathrm{MHz}$ to $900 \mathrm{MHz}$. The frequency of the $\mathrm{TM}_{010}$ mode and the cavity $\mathrm{Q}$ are measured with a network analyzer prior to each data measurement.

\subsection{Cryogenic Components}

The cavity is maintained at approximately $2 \mathrm{~K}$ by a pumped liquid helium refrigerator called the " $1 \mathrm{~K}$ pot." The $1 \mathrm{~K}$ pot uses pumping to cool helium by evaporation from $4.2 \mathrm{~K}$ at 700 Torr to less than $2 \mathrm{~K}$ at 1 Torr. The $1 \mathrm{~K}$ pot is continuously operated by feeding $4.2 \mathrm{~K}$ liquid helium into the pot through a capillary tube from a large liquid He reservoir above the cavity. Since helium is a superfluid at temperatures below approximately $2.2 \mathrm{~K}$, the pumping port of the pot has a small constriction to prevent superfluid helium from traveling up the pumping line. The $1 \mathrm{~K}$ pot is bolted directly to the top of the cavity. The noise temperature of the SQUID ("Superconducting QUantum Interference Device") amplifier depends on its physical temperature; the SQUID amplifier is cooled to near the $1 \mathrm{~K}$ pot temperature via thick copper cables between the $1 \mathrm{~K}$ pot and the SQUID amplifier package. The integration of a SQUID amplifier into ADMX is described in more detail in Ref. [1].

Lakeshore Cernox sensors measure the temperature of the ADMX insert at various locations. Calibrated sensors are mounted on the top and bottom of the cavity as well as on the SQUID amplifier package. Cernox temperature sensors are mounted on the HFET amplifiers, the vacuum can (top and bottom), bucking coil, and $1 \mathrm{~K}$ pot. Platinum sensors are installed on the insulating baffles.

\subsection{Receiver}

Figure 4 shows a schematic of the path taken by the signal from the cavity to the computer. The signal is extracted from the cavity by an electric-field probe that is nearly critically-coupled. The field probe is connected to a directional coupler used periodically to inject power into the cavity's major port to measure the coupling of the critically coupled antenna. Semi-rigid, SubMiniature version A- (SMA-) terminated, copper-jacketed RG-402 coax cables interconnect the cryogenic RF components. After the directional coupler, the signal travels through a circulator, which functions to 


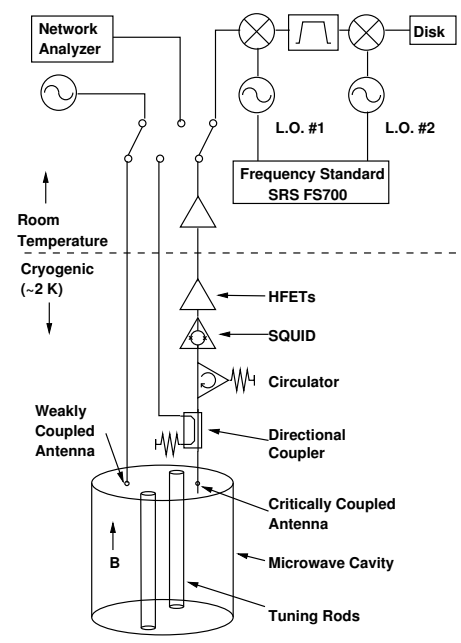

Figure 4: The architecture of the ADMX signal chain and receiver taken from Ref. [32].

impedance match to the SQUID amplifier that follows. This first-stage amplifier dominates the noise temperature of the receiver. It is followed by a HFET post-amplifier. From the cryogenic HFET postamplifier, the signal enters a rigid RG-402 transmission line that runs to the top of the ADMX insert. Outside the ADMX insert, the signal is amplified with two post-amplifiers, the last driving a 30-m Heliax cable going to signal processing hardware at the experimental site.

The first component in the signal processing rack is an Agilent 34970 switch unit. The switch allows the signal to be sent either to the network analyzer or to the first mixing stage of the receiver. The switch also allows a test signal to be sent to a weaklycoupled antenna for characterization of the cavity's resonant structure or to the directional coupler to measure the antenna coupling to the cavity. The mixing stage shifts the resonant frequency of the cavity down to $10.7 \mathrm{MHz}$ with an image-reject mixer. The down-mixed signal is then amplified by $15 \mathrm{~dB}$ and filtered by a temperature-stabilized, eight-pole, $30-\mathrm{kHz}$ bandwidth, crystal filter centered at $10.7 \mathrm{MHz}$.

After the crystal filter, the intermediate frequency signal is amplified by $15 \mathrm{~dB}$ and mixed down further to $20 \mathrm{kHz}$. An audio-frequency distribution amplifier splits the signal into 2 outputs and rejects the 21.4-MHz image signal. Two of these outputs supply analog-to-digital converters (ADCs) for voltage sampling. One of the outputs is used by an SRS 760 FFT spectrum analyzer to generate integrated power spectro 3 . The second audio signal is digitized by a National Instruments PCI-4551 card and stored for offline analysis as described later in this paper. The stored data consist of three nonoverlapping 23.8 second long time series, giving an ultimate FFT resolution of 0.042 $\mathrm{Hz}$.

\footnotetext{
${ }^{3}$ The SRS 760 FFT samples at $100 \mathrm{kHz}$ for $8 \mathrm{~ms}$, performs a fast fourier transform, computes power, and co-adds the power again until 10,000 spectra have been combined into a $50 \mathrm{kHz}$-wide, 400 -point power spectrum with Nyquist resolution $125 \mathrm{~Hz}$.
} 


\section{Data Collection}

The normal cadence of the experiment is: rotate a tuning rod to tune the cavity by a small fraction of its bandwidth (about $2 \mathrm{kHz}$ ), measure the new frequency of the $\mathrm{TM}_{010}$ mode, then digitize the down-mixed signal for approximately 80 seconds. With a receiver bandwidth of $30 \mathrm{kHz}$, an average frequency shift of $2 \mathrm{kHz}$ per tuning, and an integration time of 80 seconds, every frequency has a total exposure of approximately 1200 seconds. The experiment records data continuously until a frequency interval of $5 \mathrm{MHz}$ has been scanned with an SNR such that an axion signal would be statistically significant. After the entire 5-MHz section has the desired SNR, any given frequencies with statistically large powers are revisited and integrated continuously for 30 minutes.

The receiver center frequency is adjusted to follow the $\mathrm{TM}_{010}$ mode as the tuning rods are moved between integrations. To identify the $\mathrm{TM}_{010}$ correctly during data taking, the mode structure of the cavity is determined in advance. Before each data run a "mode map" is created by rotating a tuning rod completely around inside the cavity while the cavity swept response is measured. The $\mathrm{TM}_{010}$ mode is identified in the mode maps by comparison with computer simulations of the mode structure of the cavity for varying tuning rod positions.

The data set used in this work was collected by ADMX between May 2008 to April 2010. During this period, ADMX searched for axions in a frequency range from $860-$ $892 \mathrm{MHz}$ at approximately KSVZ axion-to-photon coupling strength. Overall, the data acquisition system generated approximately 350,000 unique spectra, each consisting of either a time series or a power spectrum, altogether totaling over 2 terabytes of raw data.

\section{Limits on Power Developed in Cavity From Axion Conversion}

The individual power spectra were combined to establish limits on the power developed in the cavity from axion conversion. This process is described in detail in Refs. [1, 32] and the steps of the process are summarized here.

The raw spectra were corrected for the response of the ADMX RF chain in two steps. First, each raw spectrum was divided by the average of all raw spectra taken on that day. This removed structure that changed very little throughout a day, mostly due to the crystal filter in the receiver. Subsequently, each spectral shape was fit with a fifth order polynomial, then divided by this polynomial. This removed the residual structure in the spectrum due to the varying response of the cavity, transmission line, and SQUID amplifier. The fifth-order polynomial was developed from the equivalent circuit model of the SQUID/receiver region and was sensitive only to structures far wider than axion signals [1, 32]. Spectra for which the fit was judged poor by a $\chi^{2}$ figure of merit were flagged as deviating from our model of receiver response and not used further. In the absence of an axion signal, the resulting corrected spectra are measurements of white noise at the system temperature. An axion signal would appear as excess power above the noise.

To combine spectra taken under different experimental conditions, the spectra are weighted by the significance of a KSVZ, $0.45 \mathrm{GeV} / \mathrm{cm}^{3}$ axion signal with power given by Eq. 3 and combined to form a "grand spectrum" spanning the entire $860-892$ 
$\mathrm{MHz}$ frequency range. The value within each bir ${ }^{4}$ of the grand spectrum was adjusted to a minimum $90 \%$ confidence level to generate limits on the power deposited in the cavity from axion conversion. The adjusted grand spectrum is used to set axion-photon coupling and axion-density limits in the next section.

\section{Limits for Specific Dark Matter Halo Models}

Limits on axion density and axion-photon coupling were calculated for each of the dark matter halo models described in Sec. 2. The grand spectrum is searched for the signal line shape predicted by each each model. Limits were set on axion-photon coupling by fixing the axion density; similarly, axion density limits were calculated by assuming the KSVZ axion-photon coupling. The axion-photon coupling limits are summarized in Fig. 5 and the axion density limits in Fig. 6. Note that the searches for both the "late infall" and the "caustic" models are sensitive only to axions moving with the specified flow velocity vector: Limits generated by these searches do not include axion flows at different velocities or zero velocity relative to ADMX. The area excluded by searches for the isothermal distribution is the generic axion exclusion limit; the other limits are highly dependent on details of the chosen halo model.

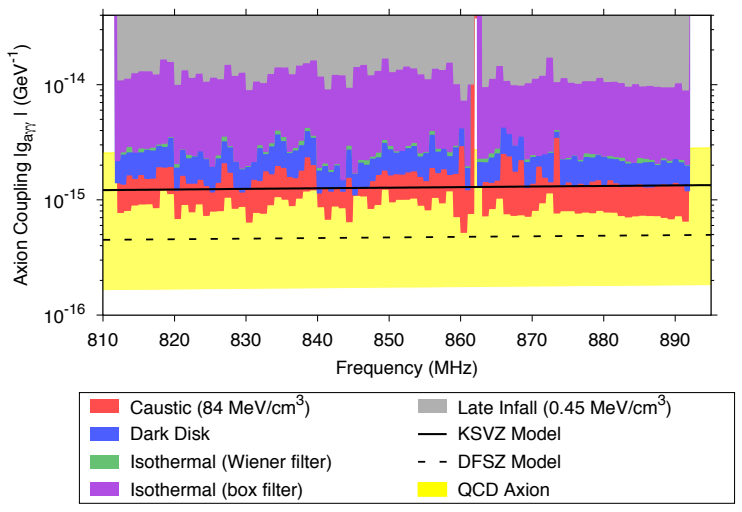

Figure 5: Limits on axion-photon coupling from $860-892 \mathrm{MHz}$ for the specific dark matter halo models detailed in the text. Each model assumes an axion density distribution and predicts the expected signal line shape. KSVZ coupling is shown as a black line $\left(\left|g_{\gamma}\right|=0.97\right)$, DFSZ coupling is shown as a dashed line $\left(\left|g_{\gamma}\right|=0.36\right)$, and the range of QCD axions is shown as a yellow band, taken from Ref. [14] as in Fig. 1] The searches for the "late infall" and "caustic" models were sensitive only to axions within each model's flow; the density of each flow is provided in the legend. The "dark disk" model includes an isothermal component and a colder disk. Limits using the isothermal distribution are to be regarded as generic axion exclusion limits; the other models are more speculative.

\footnotetext{
${ }^{4}$ Searches of axions comprising a flow (i.e. the "late infall" and "caustic" models) employed the receiver's intrinsic bin width of $0.042 \mathrm{~Hz}$; all other searches were conducted with a combined bin width of $10.74 \mathrm{~Hz}$.
} 


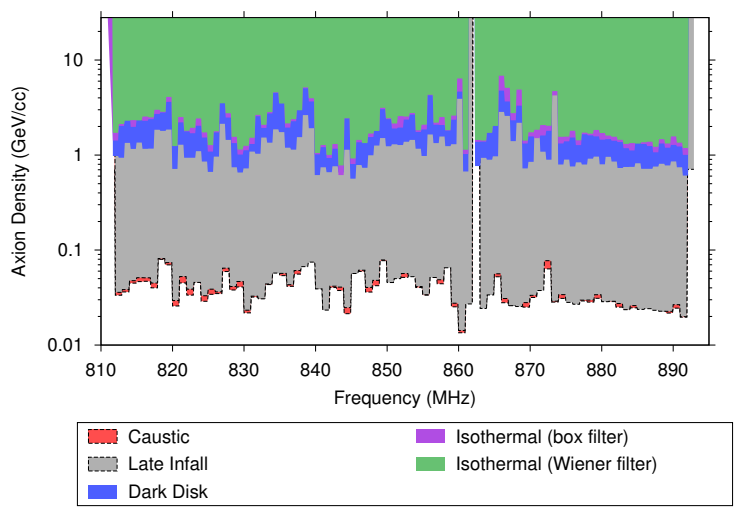

Figure 6: Limits on the local axion density from $860-892 \mathrm{MHz}$ assuming specific dark matter distribution models. All limits assume a $\left|g_{\gamma}\right|=0.97 \mathrm{KSVZ}$ axion-to-photon coupling strength from Ref. [14]. As for the coupling limits, the "isothermal" model's excluded area is the generic axion limit. The cold disk in the "dark disk" model is assumed to have 50\% the density of its isothermal component. Limits for the "dark disk" model and "isothermal" model densities for total local axion density. Limits for the "late infall" model and the "caustic" model are densities for the respective flows alone, excluding dark matter not moving at the chosen velocity.

\subsection{Isothermal Model}

The simplest model for the distribution of the dark matter in the Milky Way is an isothermal distribution [23, 24]. In this model, the total energy distribution of dark matter axions near Earth is determined by the local density of dark matter $(\rho=0.45 \pm$ $\left.0.3 \mathrm{GeV} / \mathrm{cm}^{3}\right)$, the velocity of the sun around the galaxy $\left(v_{\text {sun }}=220 \pm 20 \mathrm{~km} / \mathrm{s}\right)$, and the velocity dispersion of the dark matter at the sun's location $\left(v_{\text {disp }}=270 \pm 70 \mathrm{~km} / \mathrm{s}\right)$ [33]. This analysis used median values for $\rho, v_{\text {sun }}$ and $v_{\text {disp }}$. The resulting bandwidth is $b_{a} \simeq v_{a} \times 10^{-6}$.

A simple and robust method to search for the signal expected from the isothermal model is to apply a box filter to the grand spectrum that averages some number of adjacent bins. The use of a box filter by ADMX is described in more detail in Ref. [34]. The optimal frequency width of this filter was found analytically as follows: A synthetic axion signal was overlaid on a noise spectrum with noise uncertainty equal to the average uncertainty of the grand spectrum. Box filters of increasing width were applied to the spectrum and the resulting SNR of each was calculated. The width that maximized SNR was determined to be approximately $1 \mathrm{kHz}$.

Sensitivity was improved by exploiting the specific line shape of the isothermal model by using a modified Wiener optimal filter. The filter incorporates an expected noise power and an expected signal shape [35]. ADMX has previously employed a Wiener filter in Ref. [36]. The expected signal shape for an isothermal model is approximately a Maxwell-Boltzman distribution. The expected noise power was taken to be white. This Wiener filter yielded an improvement of 6-7\% in SNR relative to the box filter; limits generated using each filter is included in Figs. 5 and 6 . Axion density limits were generated by fixing the axion-photon coupling strength to be that of the KSVZ model. 


\subsection{Dark Disk Model}

As discussed in Sec. 2, the Milky Way may possess a disk of dark matter [26, 27]. The possible parameter space of dark disk models for the Milky Way is large, preventing an exhaustive search.

The model used in this analysis consists of an isothermal distribution with the addition of a dark disk similar to that in Ref. [37]. The isothermal distribution is identical to the model described in Sec.7.1. The dark disk is co-rotating with the Earth around the Milky Way and has a velocity dispersion of $50 \mathrm{~km} / \mathrm{s}$ and a density of $0.225 \mathrm{GeV} / \mathrm{cm}^{3}$. The expected axion signal due to the dark disk is narrower than that of the isothermal contribution by approximately a factor of 5 .

The same Wiener filter used for the isothermal model in the previous section was modified to include the expected signal from the dark disk. The additional structure of this dark disk model resulted in sensitivity to couplings 1.8 times smaller than for the isothermal distribution alone. Axion density limits were generated by fixing the axion-photon coupling to the KSVZ coupling strength as before and assuming the dark disk has 50\% density of the isothermal component; the lesser velocity dispersion of the dark disk model resulted in improved axion density limits.

\subsection{Late Infall}

Dark matter that is recently entering or exiting the Milky Way gravitational potential has not thermalized completely and is relatively cold. The expected signal width is very narrow for models including a flow of late infall dark matter and can be regarded as zero for practical purposes [30, 25]. However, there are necessary considerations to search for axions comprising a specific dark matter flow.

Namely, the motion of ADMX relative to an axion flow changes over the integration time between tunings of ADMX. The frequency shift is of the order $1 \mathrm{~Hz} /$ day, and is discussed in detail in Ref. [38]. Individual spectra that overlap in frequency may be temporally separated such that an axion signal would vary in frequency among scans. Consequently, the signal would spread out in frequency in the grand spectrum and the SNR would diminish, thereby decreasing sensitivity to axions.

One strategy to improve sensitivity to late infall axions is to choose a larger frequencybin-width such that the frequency shift of an axion signal among scans is not resolved by the analysis. This was implemented by ADMX in Ref. [2] with a bin width of 10.7 Hz. In the present analysis, however, each spectra is corrected for the specific flow velocity as follows.

The model used assumes that the halo is non-rotating, so that the infall is purely along the halo's radius. The flow has a velocity of $700 \mathrm{~km} / \mathrm{s}$ originating from a single point in the sky that is approximately opposite the galactic center $5^{5}$ the velocity is slightly greater than the local escape velocity of $544 \mathrm{~km} / \mathrm{s}$ [39]. The spectra were corrected for the changing flow velocity such that an axion signal would appear at the axion rest mass in each resulting "rest-mass" spectrum. While this procedure enhanced SNR of signals for flows at the specified velocity, this the SNR for axions at other velocities was substantially reduced.

\footnotetext{
${ }^{5}$ The location of the point origin of the flow in $(1, \mathrm{~b})$ galactic coordinates is $(150,0)$.
} 
The rest-mass spectra were then combined as before to generate a model-specific grand spectrum, and limits on axion-photon coupling strength and axion density are computed with the Wiener filter as before. The resulting axion density limits are for the density of KSVZ axions in the flow and do not include contributions from dark matter that is not moving at the chosen velocity relative to ADMX (e.g., isothermal halo).

A Monte Carlo method was used to set limits at $90 \%$ confidence as follows. The uncertainties in relative velocity between Earth and the flow are used to generate a Gaussian distribution of possible frequencies about some rest frequency. Large synthetic axion signals with frequencies sampled from the distribution are injected into the spectra prior to correction for the specific flow velocity. The spectra were then corrected for the flow velocity and combined as before. The power of these signals was reduced until $90 \%$ of the test signals are "discovered" in the grand spectrum. The power resulting in $90 \%$ discovery was taken as the $90 \%$ confidence limit. To compute axion-photon coupling limits, the density of this particular flow was fixed to $0.1 \%$ of the total halo density as in Ref. [40]. To compute limits on axion density, KSVZ coupling was chosen as before.

\subsection{Caustic Model}

In Ref. [29], Sikivie et al. argue that the late infall flows in our galaxy form dense caustic structures with nearly zero velocity dispersion. The caustic flow chosen for the present analysis is described by Duffy and Sikivie in Ref. [30] as the lesser of two flows associated with dark matter particles falling into and out of the galaxy for the fifth time ("n=5"). The inner caustic overdensity associated with the $n=5$ flows may fall near the Earth's distance from the galactic center. The chosen flow is predicted to be a nearly monoenergetic flow of dark matter axions for detection with density $84 \mathrm{MeV} / \mathrm{cm}^{3}$; this is not the flow with the highest density predicted by this model and represents a conservative choice to study sensitivity to caustic flows.

To search for this specific caustic flow, the same analysis technique as for a late infall model was used except with increased axion density and different flow velocity

relative to ADMX as predicted by Duffy and Sikivie. As with the late infall model from the previous section, the computed axion density does not include contributions from dark matter that is not moving relative to ADMX at the predicted velocity.

\section{Conclusions}

As can be seen from Figs. 5 and 6, models with finer structure or enhanced dark matter density allow ADMX to be sensitive to smaller couplings: ADMX is sensitive to axions with approximately one-half coupling strength for selected dark disk model than for the conservative isothermal distribution, and approximately one-fifth coupling strength for a caustic flow model. Conversely, once the axion is discovered, ADMX will have good discrimination capability among models by fitting the signal and comparing with line shapes predicted by various models. In general, sensitivity is greatest for the models that produce the most unique signal shapes.

At the time of this writing, ADMX is undergoing an upgrade to increase its sensitivity and scan speed significantly. It is expected that the next operation will be sensitive 
to even the most pessimistic axion couplings for any of the dark matter halo models discussed in this paper.

\section{Acknowledgements}

Supported by DOE Grants DE-FG02-97ER41029, DE-FG02-96ER40956, DE-AC5207NA27344, DE-AC03-76SF00098, the Heising-Simons Foundation, and the Lawrence Livermore National Laboratory LDRD program. SQUID development was supported by DOE grant DE-AC02-05CH11231.

[1] S. Asztalos, G. Carosi, C. Hagmann, D. Kinion, K. van Bibber, M. Hotz, L. Rosenberg, G. Rybka, J. Hoskins, J. Hwang, P. Sikivie, D. Tanner, R. Bradley, J. Clarke, "A SQUID-based microwave cavity search for dark-matter axions", Phys. Rev. Lett. 104 (2010) 041301.

[2] J. Hoskins, S. Asztalos, G. Carosi, C. Hagmann, D. Kinion, K. van Bibber, M. Hotz, L. J. Rosenberg, G. Rybka, J. Hwang, P. Sikivie, D. Tanner, R. Bradley, J. Clarke, "A search for non-virialized axionic dark matter", Phys. Rev. D 84 (2011) 121302.

[3] J. Hoskins, N. Crisosto, J. Gleason, P. Sikivie, I. Stern, N. Sullivan, D. Tanner, C. Boutan, M. Hotz, R. Khatiwada, D. Lyapustin, A. Malagon, R. Ottens, L. Rosenberg, G. Rybka, J. Sloan, D. Will, G. Carosi, D. Carter, L. Duffy, R. Bradley, J. Clarke, S. O'Kelley, K. van Bibber, E. Daw, "A modulation sensitive search for non-virialized dark-matter axions", Phys. Rev. D (in press).

[4] C. L. Bennett, M. Halpern, G. Hinshaw, N. Jarosik, A. Kogut, M. Limon, S. Meyer, L. Page, D. Spergel, G. Tucker, E. Wollack, E. Wright, C. Barnes, M. Greason, R. Hill, E. Komatsu, M. Nolta, N. Odegard, H. Peirs, L. Verde, J. Weiland, "First-year Wilkinson Microwave Anisotropy Probe (WMAP) observations: Preliminary maps and basic results", Astrophys. J. Suppl. Ser. (2003) 148 (2003) 1.

[5] V. C. Rubin, W. K. J. Ford, N. Thonnard, "Rotational properties of 21 SC galaxies with a large range of luminosities and radii, from NGC $4605(\mathrm{R}=4 \mathrm{kpc})$ to UGC 2885 (R = 122 kpc)", Astrophys. J. 238 (1980) 471.

[6] V. Trimble, "Existence and nature of dark matter in the universe", Ann. Rev. Astron. Astrophys. 25 (1987) 425.

[7] J. Ipser, P. Sikivie, "Can galactic halos be made of axions?", Phys. Rev. Lett. 50 (1983) 925.

[8] J. Preskill, M. B. Wise, F. Wilczek, "Cosmology of the invisible axion”, Phys. Lett. B 120 (1983) 127.

[9] L. Abbott, P. Sikivie, "A cosmological bound on the invisible axion", Phys. Lett. B 120 (1983) 133. 
[10] J. E. Kim, "Weak-interaction singlet and strong CP invariance", Phys. Rev. Lett. 43 (1979) 103.

[11] M. Shifman, A. Vainshtein, V. Zakharov, "Can confinement ensure natural CP invariance of strong interactions?”, Nucl. Phys. B 166 (1980) 493.

[12] A. R. Zhitnitsky, "On possible suppression of the axion-hadron interactions", Sov. J. Nucl. Phys. 31 (1980) 260.

[13] M. Dine, W. Fischler, M. Srednicki, "A simple solution to the strong CP problem with a harmless axion”, Phys. Lett. B 104 (1981) 199.

[14] J. Kim, "Constraints on very light axions from cavity experiments", Phys. Rev. D 58 (1998) 055006.

[15] R. D. Peccei, H. R. Quinn, "CP conservation in the presence of pseudoparticles", Phys. Rev. Lett. 38 (1977) 1440.

[16] S. Weinberg, “A new light boson?”, Phys. Rev. Lett. 40 (1978) 223.

[17] F. Wilczek, "Problem of strong P and T invariance in the presence of instantons", Phys. Rev. Lett. 40 (1978) 279.

[18] J. Kim, G. Carosi, "Axions and the strong CP problem", Rev. Mod. Phys. 82 (2010) 557.

[19] C. Baker, D. Doyle, P. Geltenbort, K. Green, M. van der Grinten, P. Harris, P. Iaydjiev, S. Ivanov, D. May, J. Pendlebury, J. Richardson, D. Shiers, K. Smith, "Improved experimental limit on the electric dipole moment of the neutron", Phys. Rev. Lett. 97 (2006) 131801.

[20] L. Rosenberg, "Dark-matter QCD-axion searches", Proc. Nat. Acad. Sci. 112 (2015) 12278 .

[21] P. Sikivie, "Experimental tests of the 'invisible' axion”, Phys. Rev. Lett. 51 (1983) 1415.

[22] R. Dicke, "The measurement of thermal radiation at microwave frequencies", Rev. Sci. Instrum. 17 (1946) 268.

[23] J. Navarro, C. Frenk, S. White, "The structure of cold dark matter halos", Astrophys. J. 462 (1996) 563.

[24] J. Einasto, U. Haud, "Galactic models with massive corona: I - Method. II Galaxy", Astronom. and Astrophys. 223 (1989) 89.

[25] P. Sikivie, J. Ipser, "Phase-space structure of cold dark matter halos", Phys. Lett. B 291 (1992) 288.

[26] J. Read, “The local dark matter density”, J. Phys. G: Nucl. Part. Phys. 41 (2014) 063101. 
[27] C. W. Purcell, J. S. Bullock, M. Kaplinghat, "The dark disk of the Milky Way", Astrophys. J. 703 (2009) 2275.

[28] E. Bertschinger, "Self-similar secondary infall and accretion in an Einstein-de Sitter universe", Astrophys. J. Suppl. Ser. 58 (1985) 39.

[29] P. Sikivie, I. Tkachev, Y. Wang, "The Secondary infall model of galactic halo formation and the spectrum of cold dark matter particles on earth", Phys. Rev. D 56 (1997) 1863.

[30] L. Duffy, P. Sikivie, "The caustic ring model of the Milky Way halo", Phys. Rev. D 78 (2008) 063508.

[31] M. T. Hotz, "A SQUID-Based RF Cavity Search for Dark Matter Axions", Ph.D. thesis, University of Washington (2013).

[32] S. Asztalos, G. Carosi, C. Hagmann, D. Kinion, K. van Bibber, M. Hotz, L. J. Rosenberg, G. Rybka, A. Wagner, J. Hoskins, C. Martin, N. Sullivan, D. Tanner, R. Bradley, J. Clark, "Design and performance of the ADMX SQUID-based microwave receiver”, Nucl. Instrum. Methods Phys. Res., Sect. A 656 (2011) 39.

[33] M. S. Turner, "Periodic signatures for the detection of cosmic axions", Phys. Rev. D 42 (1990) 3572.

[34] S. J. Asztalos, E. Daw, H. Peng, L. J. Rosenberg, D. B. Yu, C. Hagmann, D. Kinion, W. Stoeffl, K. van Bibber, J. LaVeigne, P. Sikivie, N. S. Sullivan, D. B. Tanner, F. Nezrick, D. M. Moltz, "Experimental constraints on the axion dark matter halo density”, Astrophys. J. Lett. 571 (2002) L27.

[35] N. Wiener, "Extrapolation, Interpolation, and Smoothing of Stationary Time Series", Wiley, 1949.

[36] S. J. Asztalos, R. F. Bradley, L. Duffy, C. Hagmann, D. Kinion, D. M. Moltz, L. J. Rosenberg, P. Sikivie, W. Stoeffl, N. S. Sullivan, D. B. Tanner, K. van Bibber, D. B. Yu, "Improved RF cavity search for halo axions", Phys. Rev. D 69 (2004) 011101.

[37] T. Bruch, J. Read, L. Baudis, G. Lake, "Signatures of the Milky Way's dark disk in current and future experiments", PoS(idm2008) 049.

[38] L. Duffy, P. Sikivie, D. Tanner, S. Asztalos, C. Hagmann, D. Kinion, L. J. Rosenberg, K. van Bibber, D. Yu, R. Bradley, "High resolution search for dark-matter axions", Phys. Rev. D 74 (2006) 012006.

[39] M. C. Smith, G. Ruchti, A. Helmi, R. Wyse, J. Fulbright, et al., "The RAVE Survey: Constraining the local galactic escape speed", Mon. Not. R. Astron. Soc. 379 (2007) 755.

[40] M. Volgelsberger, S. D. M. White, "Streams and caustics: the fine-grained structure of the $\Lambda$ CDM haloes", Mon. Not. R. Astron. Soc. 413 (2011) 1419. 